\title{
Strong magnetic fields and cosmic rays in very young galaxies
}

\author{
H. Lesch ${ }^{1}$ and M. Hanasz ${ }^{2}$ \\ ${ }^{1}$ University Observatory, München University, Scheinerstr. 1, 81679, Germany, and Center for Interdisciplinary Plasma \\ Science (CIPS) \\ e-mail: lesch@usm.uni-muenchen.de \\ 2 Toruń Centre for Astronomy, Nicholas Copernicus University, 87148 Piwnice/Toruń, Poland \\ e-mail:mhanasz@astri.uni.torun.pl
}

Received 24 September 2002 / Accepted 7 January 2003

\begin{abstract}
We present a scenario for efficient magnetization of very young galaxies about 0.5 gigayears after the Big-Bang by a cosmic ray-driven dynamo. These objects experience a phase of strong star formation during this first $10^{9}$ years. We transfer the knowledge of the connection between star formation and the production rate of cosmic rays by supernova remnants to such high redshift objects. Since the supernova rate is a direct measure for the production rate of cosmic rays we conclude that very young galaxies must be strong sources of cosmic rays. The key argument of our model is the finding that magnetic fields and cosmic rays are dynamically coupled, i.e. a strong cosmic ray source contains strong magnetic fields since the relativistic particles drive an efficient dynamo in a galaxy via their buoyancy. We construct a phenomenological model of a dynamo driven by buoyancy of cosmic rays and show that if azimuthal shearing is strong enough the dynamo amplification timescale is close to the buoyancy timescale of the order of several $10^{7} \div 10^{8} \mathrm{yr}$. We predict that young galaxies are strongly magnetized and may contribute significantly to the gamma-ray-background.
\end{abstract}

Key words. galaxies: evolution - ISM: magnetic fields - ISM: cosmic rays

\section{Introduction}

Faraday rotation measurements of high redshift objects $(z>2)$ clearly indicate that these galaxies have micro Gauss fields correlated over spatial scales of several $\mathrm{kpc}(>5 \div 10)$ at least (Athreya et al. 1998; Carilli \& Taylor 2002 and references therein). Regardless of the specifics of the Faraday screen model, the intrinsic Rotation Measures (RM) of several thousand $\mathrm{radm}^{-2}$ require such strong fields although the universe was just a sixth of its present age at redshifts $z=2.25$ and only a tenth at $z=3.8$. It is surprising that fields at such early times are similar to those found in galaxies and clusters today. These observations pose a considerable challenge to the models of magnetic field generation because of the small time available for amplifications at those redshifts, of the order of a few hundred million years. This problem has been discussed in many publications (e.g. Lesch \& Chiba 1995; Kronberg 1994 and references therein).

Here we discuss the magnetic field generation in terms of galaxy evolution at high redshifts, taking into account the consequences of the first phases of intense star formation in young galaxies. Despite many problems of the details there is now a general consensus that about half a gigayear after the Big-Bang galaxies experience a phase of strong star formation

Send offprint requests to: M. Hanasz, e-mail: mhanasz@astri.uni.torun.pl
(Steidel et al. 1999). Whereas the massive galaxies with a stellar mass at redshift $z=0$ larger than $M_{\text {Stellar }} \geq 2 \times 10^{9} h^{-1} M_{\odot}$ continue to form stars until the present epoch (Majewski 1993; Alonso-Herrero et al. 1996) ( $h$ is the Hubble constant in units of $\left.H_{0}=100 \mathrm{~km} \mathrm{~s}^{-1} \mathrm{Mpc}^{-1}\right)$ ), less massive galaxies stop forming stars at higher redshifts $(z=2 \div 3$ ) (Grebel 1997; Matteo 1998).

Blain et al. (1999) reconstruct the star formation histories compatible with the observations at various wavelengths incorporating SCUBA results (Hughes et al. 1998) and derivations from chemical evolution at high $z$. Their favorite model peaks at about $z=2$ and stays constant with higher redshift. Such a star formation history was predicted by CDM simulations (Nagamine et al. 2001). Likewise the comparison of the redshift evolution of the global star formation rate (SFR) from such simulations with the observations is in good agreement. The CDM model can explain the decreasing SFR with time. In other words, the SFR is on average a smooth function and levels off at redshifts $z>4$. This behaviour is consistent with an exponential decay of the SFR in time, on a time scale of about half a gigayear.

Very recently Lanzetta et al. (2002) addressed the effects of cosmological surface brightness dimming on observations of faint galaxies. They come to the conclusion that the incidence of the highest intensity star formation regions increases monotonically with redshift. At the very end of their paper they 
stated "Our analysis suggests that star formation in the very early universe may have occurred at a much higher rate than is generally believed and that cosmological dimming effects cannot be ignored when interpreting statistical properties of the high-redshift galaxy population".

In any case in the early phases of galaxy formation, several hundred million years after the Big-Bang the Universe was populated with intensively star forming galaxies. This is the background for our scenario about the rapid magnetization of very young galaxies via cosmic ray driven-dynamo action.

By rapid and strong magnetization we mean the amplification of galactic magnetic fields up to the equipartition values of the order of a few $\mu \mathrm{G}$ on a timescale of $10^{8} \mathrm{yr}$ starting from the beginning of star formation period in early galaxies.

Recently Zweibel (2003) evaluated the role of magnetic field strength in cosmic ray acceleration and confinement. She found that "magnetic field several orders of magnitude weaker than present galactic fields can accelerate and retain a population of relativistic cosmic rays, provided that the fields are coherent over lengthscales greater than a cosmic ray gyroradius". This result supports the point of view presented in this paper.

One can estimate that the energy output from supernovae in the form cosmic rays is more than sufficient. Assuming one $\mathrm{SN}$ per year we get $10^{8} \mathrm{SN}$ exploding within $10^{8}$ yr. Assuming that $10 \%$ of SN kinetic energy output is converted into the cosmic ray energy we obtain $10^{58}$ erg of energy released in the form of cosmic rays within $10^{8} \mathrm{yr}$.

For a typical disk volume of the order of $300 \mathrm{kpc}^{3}$, a uniform magnetic field of the strength $5 \mu \mathrm{G}$ contains $10^{55} \mathrm{ergs}$ of magnetic energy. This is 3 orders of magnitudes less than the cosmic ray energy output within $10^{8} \mathrm{yr}$. We note however that during the bursty phase of galactic evolution losses of both cosmic rays and magnetic field from galactic disks should be significant.

It is the aim of our contribution to show that the scenario of intense star formation at high redshifts has profound implications for the magnetic energy content of a galaxy. We will show that the connection between star formation and magnetic field generation and amplification is provided by the cosmic rays.

\section{Cosmic rays and the FIR-radio-correlation}

Our first subject concerns the existence of cosmic rays in galaxies. Where do they come from? What can we learn from our Galaxy and galaxies in the local universe? How can we extrapolate our findings into the early phases of galaxy evolution?

Since cosmic rays (CR) in galaxies consist mainly of protons and nuclei which do not emit much radiation, the main information about $\mathrm{CR}$ comes from their relativistic electron component which emit radio synchrotron radiation in the galactic magnetic fields. The relativistic electrons are accelerated in supernova remnants (Koyama et al. 1995; Reynolds 1996; Parizot \& Drury 2000). The observational evidence for this acceleration scenario is overwhelming since disk and starburst galaxies exhibit a very tight correlation between the spatially integrated nonthermal radio continuum flux at $6 \mathrm{~cm}$ and the thermal far-infrared (FIR)- flux at wavelengths between 40 and $120 \mu \mathrm{m}$ (Dickey \& Salpeter 1984; Helou et al. 1985;
Wunderlich et al. 1987; Wunderlich \& Klein 1988; Condon \& Broderick 1988; Völk 1989). This well-known radio-FIRcorrelation has a dispersion of only 0.2 in the logarithm of the ratio of both fluxes over more than four orders of magnitudes (Wunderlich et al. 1987).

Since the FIR emission is mainly starlight from OB-stars absorbed and reradiated by dust, the qualitative interpretation of the correlation was that the nonthermal emission in the radio band must be related to recent star formation and in fact that relativistic electrons responsible for the synchrotron emission should originate mainly in supernova remnants. Thus, the radio-FIR-correlation connects the star formation and magnetized cosmic rays by particle acceleration in supernova remnants. This is the first piece of evidence that star formation is accompanied by intense cosmic ray production.

A second piece of evidence was presented by Völk et al. (1989). They argued on the basis of the spallation age of galactic cosmic rays and the radio luminosity that the supernova remnants are the main sources for the cosmic rays. They applied this argument to the starburst galaxy M 82 and our Galaxy and found the very surprising result that for both galaxies the simple relation holds that the cosmic ray production rate $\dot{E}_{\mathrm{CR}}$ is proportional to the supernova rate $f_{\mathrm{SN}}: \dot{E}_{\mathrm{CR}} \propto f_{\mathrm{SN}}$. This is a surprise since both galaxies are completely different with respect to their cosmic ray energy density, magnetic energy density and supernova rate $f_{\mathrm{SN}}$ (in our Galaxy $f_{\mathrm{SN}}=(30 \mathrm{yr})^{-1}$, whereas in M $82 f_{\mathrm{SN}} \simeq(1 \mathrm{yr})^{-1}$, Schaaf et al. 1989). Especially cosmic ray and magnetic energy density differ by two orders of magnitudes, in M 82 they are 100 times larger than in our Galaxy. Völk et al. (1989) concluded that their simple scaling should be applicable for all kinds of galaxies. In other words, in all galaxies, no matter what evolutionary state they have reached the supernova rate is a direct measure for the cosmic ray production rate.

The total cosmic ray injection power per unit volume is

$\frac{\mathrm{d} W_{\mathrm{CR}}}{\mathrm{d} t}=\langle E q\rangle V=\frac{\langle E q\rangle}{\bar{\rho}} \bar{M}$,

where $\bar{M}=\bar{\rho} V$ is the gas mass contained in the cosmic ray propagation volume $V .\langle E q\rangle$ denotes the cosmic ray injection power per unit volume

$\langle E q\rangle=A \int_{0}^{\infty} E q_{E} \mathrm{~d} E$,

with the number $q_{E}$ of accelerated particles per unit volume, unit time and unit energy band. The mass number $A$ accounts for the fact that $E$ is the energy per nucleon. Although the cosmic ray power depends on the source spectrum via $q_{E}$, on the gas mass and the propagation volume, different authors find quite an agreement in absolute numbers. Drury et al. (1989) as well as Fields et al. (2001) found a total cosmic ray power for the Galaxy

$\frac{\mathrm{d} W_{\mathrm{CR}}}{\mathrm{d} t}=1.1-1.5 \times 10^{41} \mathrm{erg} \mathrm{s}^{-1}$

using an injection spectrum proportional to $p^{-2.2}$ taken from the observations. 
As mentioned above there is no doubt that shock waves originating from supernova explosions play a major role for the acceleration of cosmic rays (for electron acceleration to energies above $100 \mathrm{TeV}$ see e.g. Koyama et al. 1995; Tanimori et al. 1998, for direct evidence of nucleon acceleration to $E>$ $300 \mathrm{MeV} /$ nucleon see Combi et al. 1998). The net Galactic cosmic ray injection power due to supernovae $\frac{\mathrm{d} W_{\mathrm{SN}}}{\mathrm{d} t}$ is proportional to the supernova rate and average mechanical energy output of a supernova times some efficiency which is transformed into cosmic rays. Since the supernova rate is proportional to the star formation rate $\frac{\mathrm{d} W_{\mathrm{SF}}}{\mathrm{d} t}$ one finally ends up with a scaling that $\frac{\mathrm{d} W_{\mathrm{SN}}}{\mathrm{d} t} \propto \frac{\mathrm{d} W_{\mathrm{SF}}}{\mathrm{d} t}$. Fields et al. (2001) derived this intuitive scaling from quantitative arguments by a careful and consistent model for the energetics of cosmic ray acceleration and the abundances of $\mathrm{LiBeB}$, which are due to cosmic ray nucleosynthesis in the interstellar medium. Doing so, they could go a step further and they derive an even more direct scaling of the star formation rate and the cosmic ray acceleration rate, as has been implicitly assumed by most previous work on early Galactic cosmic ray nucleosynthesis (see next section).

Here we can summarize that beyond any doubt the rate at which stars are formed in a galaxy is a direct measure for the cosmic ray flux.

\section{Arguments from the scenario of Galactic cosmic ray nucleosynthesis}

An interesting side-line of our scenario concerns the observations of Be and B in old stars of the Milky Way halo (Gilmore et al. 1992; Duncan et al. 1992). B and Be are spallation products of cosmic ray protons colliding with $\mathrm{C}, \mathrm{N}$ and $\mathrm{O}$. The observations prove that these elements were present in the early Galaxy in amounts much larger than conventional scenarios of nucleosynthesis can account for. The B/Be-ratio is, however, quite consistent with the Galactic cosmic rays (GCR) nucleosynthesis scenario. In order to explain this intriguing results Prantzos et al. (1993) and Prantzos \& Casse (1995) showed that one solution is that during the early phase of the Galaxy GCR were more efficiently confined than today. As a result very high GCR fluxes were obtained. If this is a generic feature of the early phases of galaxy evolution, large gamma-ray fluxes resulting from pion decay should have been produced at redshifts $z>3 \div 10$. Prantzos \& Casse also found that the GCR in the early phases of Galaxy evolution must have been more confined to the Galaxy than today to establish the B/Be ratio. This means that something kept the GCR closer to the Galaxy as today. This can only be provided by a stronger magnetic field and/or a higher gas density. Latter is reasonably to expect in young galaxies. Both components are acting on the CR. It is currently thought that GCR are accelerated mainly by supernovae shock waves and propagate in the Galaxy mainly by diffusing on the irregularities of the Galactic magnetic field, thereby suffering energy losses by ionization, nuclear reactions and leakage in the framework of the "leaky box" model (e.g., Cesarsky 1980). A higher confinement at earlier phases than means that the box was "closed" rather than a "leaky" box for GCRs (Prantzos \& Casse 1995). The component of the interstellar medium which can hinder the GCR to escape freely is the magnetic field.

This requirement must be understood in terms of a significantly higher supernova rate than today, since Parizot \& Drury (2000) showed that the elements B and Be can be produced in the early Galaxy only, by the combined action of collective supernova exploding in OB-associations driving expanding superbubbles. That is, the high amount of B and Be in the old stars tells us that many more supernova explosions must have been occurred at an early evolutionary stage of the Galaxy to provide these elements. Or simply, that the supernova rate at the early stages was much higher than today. If we combine these results with those from Völk et al. (1989) the physical origin of an enhanced CR-flux in the early phases of the Galaxy has to be a higher supernova rate and a higher magnetic field strength than today. Somehow there must be a dynamical coupling between the supernova remnant shock waves the $\mathrm{CR}$ and the magnetic field strength.

\section{Cosmic rays and dynamo action}

Thus, we now introduce the connection of the magnetic field to the radio-FIR-correlation, which was found by Lisenfeld et al. (1996). They presented an analysis of the radio-FIR-correlation in starburst galaxies in comparison to a control sample of normal spiral galaxies. Here we cite their main conclusions:

- The mean FIR to radio ratio showed no significant difference between the starburst sample and the control sample and is independent of the starburst strength.

- The magnetic field is required to be amplified strongly on short time-scales $\left(\sim 10^{7} \mathrm{yr}\right)$ in order to maintain a sufficiently constant FIR to radio flux ratio in the first $2 \times 10^{7} \mathrm{yr}$ of a starburst. Otherwise the inverse Compton losses during this phase would lead to a very low synchrotron emission, resulting in a too large value of the FIR to radio flux ratio, significantly higher than the observed one.

What can we learn from these findings for the high redshift objects? We can learn that an intense starburst must produce lots of CR and must produce strong magnetic fields to confine the $\mathrm{CR}$ to explain the B/Be-ratio. We know the sources for the cosmic rays - the supernova remnants. What is the source for the field amplification? Our answer is: the CR population.

In the Milky Way the radial distribution of the molecular clouds where stars are born, the distribution of supernova remnants and the distribution of cosmic rays are different. The column density of molecular gas comprises a peak at the Galactic Center and a peak at the galactocentric radius $R_{\mathrm{G}}=4.5 \mathrm{kpc}$. Behind $R_{\mathrm{G}}=10 \mathrm{kpc}$ the column density drops out below a few per cent of its peak value (Clemens et al. 1988). The supernova distribution has a peak at $4.5 \mathrm{kpc}$ and extends up to $14 \mathrm{kpc}$ (Case \& Bhattacharya 1998). The galactic cosmic rays have a considerably broader distribution up to $20-30 \mathrm{kpc}$ in galactic radius (Strong \& Moskalenko 1998). The latter distribution suggests the existence of a self-regulation (valve) mechanism which removes the excess of cosmic rays above certain critical level of cosmic ray energy density (see Hanasz \& Lesch 2001; Ptuskin 2001). This supports the model of Breitschwerdt et al. (1993) who showed the possibility of an intense cosmic 
ray-driven galactic wind if the CR-energy density exceeds a critical value.

The supernovae influence the dynamics of ISM in multiple ways through the input of kinetic energy, thermal energy and cosmic ray energy to the surrounding medium. The kinetic energy injected in a certain spatial scale cascades to both smaller and larger scales in the form of turbulent cascade. The thermal energy resulting from the shock heating produces the X-ray emission of ISM and leads to other observable effects like supernova remnants, superbubbles, galactic chimneys (Korpi et al. 1999) and galactic fountains (Kahn \& Brett 1993)

Cosmic rays are dynamically important for the ISM because their energy density is comparable to the energy densities of the remaining ingredients of ISM. This means that on average cosmic rays contribute to the pressure which is comparable to the pressure of thermal gas (including the thermal and turbulent pressure) and to the pressure of magnetic field (see e.g. Ferriere 1998a for a detailed model of the Galactic ISM). An additional factor essential for the distribution of various ISM ingredients in galactic disks is gravity, which comes partially from the mass of stars, dark matter and the thermal gas itself. One should note that cosmic rays and magnetic field are weightless components. Cosmic ray particles provide a significant pressure to the multi-component interstellar medium even though their total mass in negligible (1 cosmic ray nucleon per $10^{9} \mathrm{~cm}^{3}$ ). Since they contribute to the total pressure but do not contribute to the mass density of ISM, they support partially the heavy interstellar gas from below. Such a configuration is known to be unstable against the buoyancy instability which is known as Parker instability. The Parker instability operates in magnetized, gravitationally stratified atmospheres (Parker 1966, 1967) and is a magnetic version of the RayleighTaylor instability.

All the three chains of supernova energy conversion are potential sources of magnetic field amplification, however the efficiency of each chain is different.

The kinetic energy is supposed to contribute to the cascade of helical turbulence which plays a central role in the classical mean field dynamo theory. About 10 percent of SN energy is converted to turbulent energy (Dorfi 1993). The hot gas contributes to the dynamo $\alpha$-effect through the expansion of supernova remnants and superbubbles (Ferriere 1998b), or through galactic fountains (Kahn \& Brett 1993). Although majority of the SN energy becomes thermal energy at some stage of SNR evolution, there are two chains of losses of the thermal gas. The first one is cooling of the hot interstellar gas, the second is the escape of the light and buoyant hot gas from galactic disks in chimney flows.

However, the hot gas fills only less than $20 \%$ of the present day Milky Way ISM volume (Ferriere 2001), mostly in hot bubbles. The hot bubbles are supposed to be the source for the $\alpha$-effect in conventional dynamos (Ferriere \& Schmidt 2000), which are by a factor of 10 too slow for the amplification of the regular magnetic field component (Lesch \& Camenzind 1994; Hanasz \& Lesch 2001).

On the other hand, cosmic rays are much more efficient since they fill the whole galaxies (due to the diffusive propagation) and couple dynamically to the magnetic field. The dynamical coupling of the cosmic ray component and magnetic field follows from the fact that cosmic ray particles can propagate only along magnetic field lines (the gyroradius of typical $1 \mathrm{GeV}$ protons in the $\mu \mathrm{G}$ magnetic field is of the order of $10^{13} \mathrm{~cm}$, which is 8 orders of magnitude below the typical galactic spatial scales. Moreover, the bulk propagation of cosmic rays along magnetic field lines is slowed down much below the speed of light. Due to the streaming instability cosmic rays excite turbulence (Kulsrud \& Pearce 1969). The turbulence scatter cosmic ray particles back and forth and the bulk motion of relativistic cosmic ray gas is slowed down to the Alfvén speed, which is typically a few tens $\mathrm{km} \mathrm{s}^{-1}$.

Parker (1992) proposed a new kind of galactic dynamo powered by the very powerful output of cosmic rays accelerated in supernova remnants. That kind of fast dynamo incorporating the buoyancy of cosmic rays, Coriolis force, differential rotation and magnetic reconnection is expected to amplify the large scale magnetic field in timescales $\leq 10^{8} \mathrm{yr}$ or even faster. We are going to demonstrate in the next section that the amplification timescale of the cosmic ray driven dynamo is indeed related to the buoyancy timescale, which is of the order of $\leq 10^{8} \mathrm{yr}$.

It was demonstrated by Hanasz \& Lesch (2000) that the Parker instability excited by the cosmic ray supply in supernova remnants becomes nonlinear immediately after explosion, resulting in a significant acceleration of the Parker instability above the standards of the linear approximation. The same circumstance - nonlinearity since the very beginning implies that all the linear instability criteria become invalid. The Parker (buoyancy) instability is usually considered as a destructive effect for large scale galactic magnetic fields (see Hanasz \& Lesch 2001), however in the presence of Coriolis force, differential rotation and magnetic reconnection cosmic rays provide a means for a very efficient amplification of the large scale magnetic field.

The idea of Parker's (1992) fast galactic dynamo is based on the sequence of following processes. The primary driver of this kind of dynamo in galactic disks is the powerful supply of cosmic rays in supernova remnants. The cosmic rays inflate lobes on the magnetic field. The magnetic lobes extending up to the galactic halo are tightly packed and form tangential discontinuities in magnetic field. Then fast magnetic reconnection starts to change the topology of magnetic field forming either closed magnetic loops as it is proposed in the original Parker's scenario, or with the aid of Coriolis force, helical tubes of magnetic field. (We note following Parker that the term fast dynamo is due to the contribution of fast magnetic reconnection, which provides the necessary dissipation of the small scale magnetic field). The latter possibility has been demonstrated by means of numerical simulation by Hanasz et al. (2002). The further reconnection of loops (which undergo rotation caused by Coriolis force) or helical tubes leads to the production of the radial component of magnetic field. The new radial magnetic field is subsequently stretched by differential rotation, leading to the amplification of the toroidal component of magnetic field. 


\section{Phenomenological model of the buoyancy driven dynamo}

In the present section we construct a phenomenological buoyancy-driven dynamo model resulting from our previous investigations (Hanasz \& Lesch 1998, 2000, 2002; Hanasz et al. 2002). The buoyancy (Parker) instability operates in the absence of cosmic rays, however if cosmic rays are additionally present in the system, the buoyancy timescale shrinks significantly. Investigations of buoyancy driven galactic dynamos were performed also by other authors (Moss et al. 1999) incorporate two modifications to the standard dynamo equation. First, they assume that the dynamo $\alpha$ parameter is an increasing function of magnetic field strength and second, that the large-scale rotation velocity is supplemented by a regular outflow velocity due to buoyancy, however they did not take into account cosmic rays. In their investigations efficient amplification of the large-scale magnetic field is possible if magnetic field responsible for buoyanacy is strong enough. In the current model buoyancy is provided by both magnetic field and cosmic rays, so the strength of magnetic field is not crucial. Our model relies on the following three elements:

1. In the absence of rotation, buoyancy of the magnetic field and cosmic rays evacuates the magnetic field from the disk on a timescale of a few $100 \mathrm{Myr}$, without the cosmic ray contribution, down to a few 10 of Myr when the Parker instability is driven by cosmic ray injection in supernova remnants. We define the buoyancy timescale $t_{\text {buo }}$ as the characteristic time of removal of the magnetic field via Poynting flux (instead of relating it to the growth time of Parker instability in the linear regime). In our model the vertical Poynting flux replaces the concept of turbulent diffusivity in classical turbulent dynamos.

2. It follows from a linear analysis of Parker instability and from numerical simulations (Hanasz \& Lesch 1998) that the instability leads to the formation of kpc-scale helices on initially horizontal magnetic field lines. This finding is a modification of Parker's (1992) original scenario involving the dynamics of closed magnetic loops. The formation of helices, which result from the action of the Coriolis force and tidal forces in differentially rotating disks, is equivalent to the production of a negative radial magnetic field component in lower layers of the disk and a positive magnetic field component in upper layers of the disk. This scenario has been confirmed by numerical simulations presented in recent papers (Hanasz \& Lesch 2000, 2002; Hanasz et al. 2002). Hanasz \& Lesch (1998) have shown that the production of the radial magnetic field component in the helices is equivalent to the presence of the dynamo $\alpha$-effect. The neighboring helices merge as a result of fast magnetic reconnection, producing a large-scale poloidal magnetic field. The essential point of our argument is that, according to numerical simulations, in the presence of realistic (rigid) rotation, the radial magnetic field of the significant magnitude of $10 \div 30 \%$ (larger and smaller values are possible) of the azimuthal component is built within the buoyancy timescale $t_{\text {buo }}$.
3. We take into account differential rotation which produces the azimuthal magnetic field from the poloidal one. The differential rotation enters our system through the shearing parameter $G=r \mathrm{~d} \Omega / \mathrm{d} r$. The timescale of generation of an azimuthal magnetic field from a poloidal one is $t_{\mathrm{G}}=1 /|G|$.

Assuming a thin disk geometry, the superposition of all three mentioned effects can be expressed by the following set of equations describing evolution of the mean magnetic field $B_{\mathrm{r}}$ and $B_{\varphi}$ in the disk

$$
\begin{aligned}
& \frac{\mathrm{d} B_{\mathrm{r}}}{\mathrm{d} t}=-\frac{C_{\Omega}}{t_{\text {buo }}} B_{\varphi}-\frac{1}{t_{\text {buo }}} B_{\mathrm{r}}, \\
& \frac{\mathrm{d} B_{\varphi}}{\mathrm{d} t}=-\frac{1}{t_{\mathrm{G}}} B_{\mathrm{r}}+\frac{C_{\Omega}}{t_{\text {buo }}} B_{\mathrm{r}}-\frac{1}{t_{\text {buo }}} B_{\varphi},
\end{aligned}
$$

where $C_{\Omega} \sim 0.1 \div 0.3$ is a constant factor representing our expectation that radial magnetic field of the order of $0.1 \div 0.3$ of the azimuthal magnetic field strength is efficiently produced within the timescale $t_{\text {buo }}$. The constant factors $\pm C_{\Omega}$ are related to the dynamo $\alpha$ parameter as described by Hanasz \& Lesch (1998).

The present dynamo concept relies on the assumption that fast magnetic reconnection efficiently merges neighboring helices and produces a new large-scale poloidal magnetic field of opposite sign in the lower half and the upper half of the disk thickness $H$. The mean vertical buoyant velocity removes the magnetic field from the upper layer of the disk volume. After $t_{\text {buo }}$ only the magnetic field from the lower layer remains within the disk volume.

The negative sign in front of $C_{\Omega}$ in Eq. (4) and positive sign in front of $C_{\Omega}$ in Eq. (5) are justified by the fact that the lower (remaining) parts of spirals are twisted by the Coriolis force in the direction of galactic rotation while the upper (rejected) parts are twisted against the galactic rotation (see Figs. 1 and 2 in Hanasz \& Lesch 1998).

We search for harmonic solutions of the system of Eqs. (4) and (5) through the substitution

$\left(\begin{array}{c}B_{\mathrm{r}} \\ B_{\varphi}\end{array}\right)=\left(\begin{array}{c}B_{\mathrm{r} 0} \\ B_{\varphi 0}\end{array}\right) \exp (-i \omega t)$

and obtain the following equation for the complex oscillation frequency:

$\omega^{2}+\frac{2 i}{t_{\text {buo }}} \omega+\frac{C_{\Omega}}{t_{\mathrm{G}} t_{\text {buo }}}-\frac{C_{\Omega}^{2}}{t_{\text {buo }}^{2}}-\frac{1}{t_{\text {buo }}^{2}}=0$.

Complex solutions of the above second order polynomial equation are

$\omega_{ \pm}=\frac{1}{t_{\text {buo }}}\left[-i \pm\left(C_{\Omega}^{2}-C_{\Omega} \frac{t_{\text {buo }}}{t_{\mathrm{G}}}\right)^{1 / 2}\right]$.

The real part of $\omega$ is the oscillation frequency and the imaginary part represents the growth rate of the large-scale magnetic field. We note that in the absence of rotation, only a damped nonoscillating solution is present, ie. the growth rate is $\operatorname{Im}(\omega)=$ $-1 / t_{\text {buo }}$. If rigid rotation $\left(t_{\mathrm{G}}=0\right)$ is present then the real part of $\omega$ becomes non-vanishing and the damped solutions oscillate 


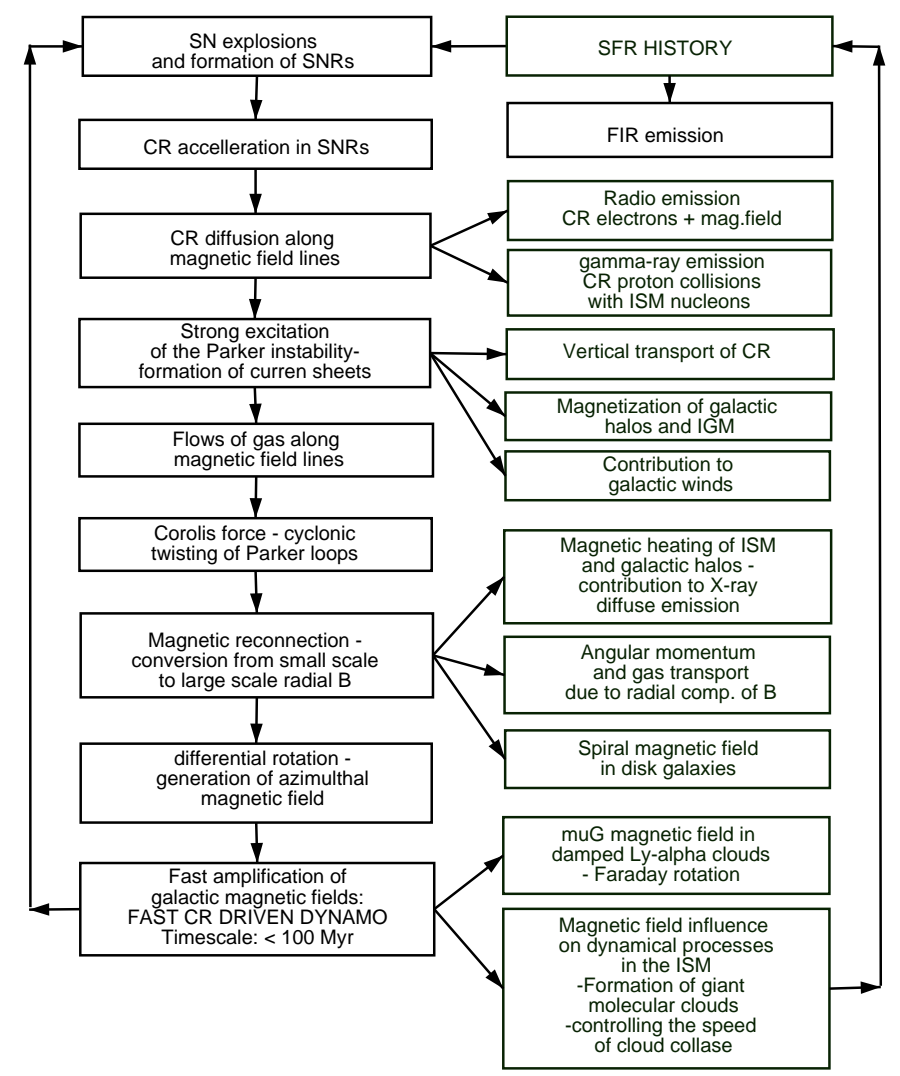

Fig. 1. Flow diagram of the cosmic ray driven fast dynamo and related phenomena.

with frequency $\operatorname{Re}(\omega)= \pm C_{\Omega} / t_{\text {buo }}$. If in addition differential rotation is present and is strong enough $\left(t_{\mathrm{G}} \leq t_{\text {buo }} / C_{\Omega}\right)$ then solutions become non-oscillatory and the rotation contributes to the imaginary part of $\omega$.

Amplification of the mean magnetic field is possible when $\operatorname{Im}\left(\omega_{+}\right)>0$. The condition for the existence of growing solutions can be expressed as

$t_{\mathrm{G}}<\frac{t_{\text {buo }}}{C_{\Omega}+\frac{1}{C_{\Omega}}}$.

The factor $1 /\left(C_{\Omega}+1 / C_{\Omega}\right)$ reaches its maximum value of 0.5 at $C_{\Omega}=1$ so we deduce that the weakest restriction for the shearing timescale coming around $C_{\Omega}=1$ is

$t_{\mathrm{G}}<0.5 t_{\text {buo }}$

We note that realistic present day galactic disks seem to fulfill this relation, e.g. in the Milky Way $t_{\mathrm{G}} \simeq 15-30 \mathrm{Myr}$ for galactocentric radii ranging from $0.5 R_{\odot}$ to $1 R_{\odot}, t_{\text {buo }}$ is perhaps $50 \div 100 \mathrm{Myr}$.

If $C_{\Omega}<1$ then we find that amplification is possible if $t_{\mathrm{G}}<t_{\text {buo }} / 2.5$ for $C_{\Omega}=0.5, t_{\mathrm{G}}<t_{\text {buo }} / 3.5$ for $C_{\Omega}=0.3$ and $t_{\mathrm{G}}<t_{\text {buo }} / 10$ for $C_{\Omega}=0.1$. The limitation (10) for the shearing timescale seems to be quite restrictive, however, different origins of shearing motions can be considered: the galactic differential rotation, the shearing resulting from the presence of galactic bars, density waves as well as galactic close encounters.
The most essential property of our model is that if only the amplification condition (9) is fulfilled, the amplification of the mean magnetic field proceeds on a timescale which is close to the buoyancy timescale. The buoyancy timescale $t_{\text {buo }}$ is, on the other hand, controlled by the production rate of cosmic rays. The presented dynamo model directly relates the dynamo amplification timescale $t_{\mathrm{D}}$

$t_{\mathrm{D}}=\frac{1}{\omega_{+}}=t_{\text {buo }}\left[\left(C_{\Omega} \frac{t_{\text {buo }}}{t_{\mathrm{G}}}-C_{\Omega}^{2}\right)^{1 / 2}-1\right]^{-1}$

to the production rate of cosmic rays. Although it is difficult to precisely quantify this relation, we can say that the buoyancy timescale is at least an order of magnitude shorter than the corresponding turbulent diffusion timescale controlling the classical dynamos; thus, we deduce that the cosmic ray-driven dynamo is an order of magnitude faster than the classical turbulent dynamos.

We expect therefore that the dynamo amplification timescale $t_{\mathrm{D}}$ may be very short in galaxies intensely producing cosmic rays; however the amplification condition requires that the shearing timescale $t_{\mathrm{G}}$ is a few times shorter than $t_{\text {buo }}$. Finally, we note that the present phenomenological model is far from precise, although it seems to display correctly the basic properties and role of different timescales in our dynamo model.

\section{Implications}

The whole process forming a closed cycle is expected to amplify the large-scale galactic magnetic field on a time scale $t_{\mathrm{D}} \sim 10^{8} \mathrm{yr}$ as deduced for the present-day galaxies like our Milky Way. The fast dynamo process involves magnetic reconnection serving as a dissipative process converting the smallerscale turbulent field into the large scale field, so the turbulent field has to form first within a few $10^{7} \mathrm{yr}$. The latter timescale corresponds to the growth time of magnetic buoyancy instability. The detailed field structure seems to be not very crucial to explain the findings by Lisenfeld et al. (1996); however the field structure is decisive for the cosmic rays transport (see e.g. Giacalone \& Jokipii 1999) and the onset of Parker instability (Parker \& Jokipii 2000; Hanasz \& Lesch 2000). Strong organized magnetic fields are observed in the radio halo of M 82 (Reuter et al. 1992) and in compact dwarf galaxies (Klein et al. 1991). Except in the nuclear region of M 82 the magnetic field is horizontal in significant parts of the disk of that galaxy (Reuter et al. 1992). This confirms the link between the turbulent and the large-scale magnetic fields in starburst galaxies.

Due to the significantly higher star formation rate in early galaxies we expect that the strength of the primary driver - the cosmic ray production-rate was much larger in the past leading to even faster amplification of galactic magnetic fields. We present the idea of a fast cosmic ray driven dynamo in the left part of diagram in Fig. 1.

In the right part of the diagram we point out various effects that are physically related to the fast dynamo cycle. First, the star formation history is the input parameter for the cosmic ray driven dynamo. The stellar evolution leads to the production of dust in the interstellar medium, which is the source of 
the FIR radiation. Second, the direct implication of the presence of cosmic rays is the radio synchrotron emission from cosmic ray electrons gyrating in the galactic magnetic field and the gamma-ray emission coming from collisions of CR protons against ISM nucleons.

Third, the direct consequence of the cosmic ray driven Parker instability is vertical transport of ISM components, primarily cosmic rays which cumulate at tops of rising Parker loops (see Fig. 3 of Hanasz \& Lesch 2000). The vertical buoyant motions influence also the magnetic field which is very efficiently removed from the disk by the Parker instability (see Fig. 8 of Hanasz et al. 2002). The buoyant rise of magnetic lobes and cosmic rays push gas just above, leading to so-called "helmet flows", resembling the familiar bursts of solar wind.

Fourth, magnetic reconnection converts the small-scale helical structures coherently twisted by the Coriolis force into the large-scale spiral magnetic field. The horizontal alignment of the resulting field forms the condition for the next outburst of the Parker instability. Moreover, magnetic heating of the ISM and galactic halo has been proposed by Parker (1992) and further investigated by Hanasz \& Lesch (1998) and other authors (Tanuma et al. 2003). The presence of a radial magnetic field has to give rise to some angular momentum transport in galactic disks on cosmological timescales. The reconnection and topological evolution of the distorted magnetic field forms specific conditions for cosmic ray propagation. As we pointed out already, the trajectories of cosmic ray particles are determined by the shape of magnetic field lines, therefore after the onset of the Parker instability cosmic rays are released from the disk (the vertical growth of magnetic loops with cosmic rays does not stop) and after the reconnection/relaxation of the magnetic field structure, cosmic rays are confined in the disk by the magnetic field.

Fifth, the fast magnetic field amplification offered by the fast dynamo model provides a chance to explain the $\mu \mathrm{G}$ magnetic field in early galaxies (Wolfe et al. 1992).

Often it is stated that in radio galaxies at high redshifts the active radio source itself magnetizes the ambient thermal plasma. This was discussed by Athreya et al. (1998) in their conclusions: the synchrotron plasmas in the radio lobes are permeated by strong magnetic fields of hundreds of $\mu \mathrm{G}$ or even a magnitude higher in the active hotspot, especially in powerful radio sources. The magnetic field is supposed to be effectively transported by the radio jet from the radio nucleus. Turbulent diffusion and magnetohydrodynamical instabilities may lead to such magnetic field transport into the surrounding thermal plasma (Bicknell et al. 1990). However, there is no observational evidence for such transport. The observations show almost no mixing between the synchrotron plasma and the thermal plasma, especially near the hotspot where the two plasmas are well separated by the bow shock (Carilli et al. 1994). It is believed that the radio jets evacuate the cluster medium along their paths with very little mixing of the two media. A similar effect is visible in the galaxy $0902+343(z=3.4)$ with its line-emitting gas anticorrelating with the radio source (Carilli 1995). Even theoretically it is not clear if the hotspot field can be diffused into the region around it on a timescale fast enough to produce a deep Faraday screen surrounding the hotspot before it moves ahead substantially (i.e. within $10^{6}$ years).

Accordingly, one must investigate alternative mechanisms for the strong magnetic fields at high redshifts indicated by the Faraday observations (e.g. Athreya 1998).

Finally, a magnetic field efficiently amplified during early galactic evolution has to influence the various dynamical processes in galactic disks. The coupling of Parker (buoyancy), Jeans (gravitational) and Field (thermal) instabilities leads to the formation of giant molecular clouds (Blitz \& Shu 1980). On the other hand the collapse of the cloud cores is expected to be slowed down by the presence of magnetic pressure. The interplay of these two processes provides a kind of self-regulation mechanism which may influence the star formation rate and the initial mass function (IMF) (Rees 1994). The star formation rate is the input parameter for our fast dynamo, thus the interplay of these processes controls the efficiency of the cosmic ray driven dynamo.

\section{Conclusions}

All observations indicate that young galaxies are characterized by phases of strong star formation. The radio-far-infraredcorrelation observed in numerous galaxies with completely different star formation rates proves that the formation of stars is accompanied by efficient production of cosmic rays. In other words, a strong starburst is equivalent to strong magnetic fields generated within a few $10^{7} \div 10^{8}$ years and an intense cosmic ray flux. Observations of old stars in the halo of the Milky Way supports this picture. They contain significant amounts of spallation products (B and $\mathrm{Be}$ ) which are due to a cosmic ray flux in the young Galaxy which was considerably higher than today. On top of that, the cosmic ray propagation at these early phases of galactic evolution can only be understood if the magnetic field strengths were higher than its current values.

If we transfer these findings to young galaxies we expect that these objects contain many more cosmic rays than fully evolved galaxies. In a series of papers, we have shown that cosmic rays can drive an efficient galactic dynamo based on outflows from the star formation regions. The advantage of the CR-dynamo is twofold: first, it is very fast on time scales of a few $10^{7} \div 10^{8}$ years, whereas conventional dynamos amplify the disk field on timescales of gigayears (Camenzind \& Lesch 1994). Second, the CR-dynamo thrives on the outflows produced by star formation activity, whereas conventional dynamos are susceptible to inevitable disruption by outflows. The stronger the disk activity, the faster and more effective is the CR-dynamo.

Thus, very young galaxies are strongly magnetized. We predict that observations even at very high redshifts $(z>4)$ will find many galaxies with high radio fluxes.

Acknowledgements. We deeply thank Heinz Völk for insightful discussions and very helpful advices. We thank the referee L. O. C. Drury for his comments which improved the paper. This work was supported by the Polish Committee for Scientific Research (KBN) through the grant PB PB 404/P03/2001/20. The presented work is continuation of a research program realized by $\mathrm{MH}$ under the financial support of Alexander von Humboldt Foundation. 


\section{References}

Alonso-Herrero, A., Aragon-Salamanca, A., Zamorano, J., \& Rego, M. 1996, MNRAS, 278, 417

Athreya, R. M., Kapahi, V. K., Mc Carthy, P. J., \& van Breugel 1998, A\&A, 329, 809

Bicknell, G. V., Cameron, R. A., \& Gingold, R. A. 1990, ApJ, 357, 373

Blain, A. W., Smail, I., Ivison, R. J., \& Kneib, J.-P. 1999, MNRAS, 302,632

Blitz, L., \& Shu, F. H. 1980, ApJ, 238, 148

Camenzind, M., \& Lesch, H. 1994, A\&A, 284, 411

Carilli, C. L., Owen, F. N., \& Harris, D. E. 1994, AJ, 107, 480

Carilli, C. L. 1995, A\&A, 298, 77

Carilli, C. L., \& Taylor, R. B. 2002, ARA\&A, 40

Case, G. L., \& Bhattacharja, D. 1998, ApJ, 504, 761

Clemens, D. P., Sanders, D. B., \& Scoville, N. Z. 1988, ApJ, 327, 139

Cesarsky, C. 1980, ARA\&A, 18, 289

Combi, J. A., Romero, G. E., \& Benaglia, P. 1998, A\&A, 333, L91

Condon, J. J., \& Broderick, J. J. 1988, ApJS, 96, 30

Dickey, J. M., \& Salpeter, E. E. 1984, ApJ, 284, 461

Drury, L. O., Markiewicz, W. J., \& Völk, H. J. 1989, A\&A, 225, 179

Duncan, D., Lambert, D., \& Lemke, D. 1992, ApJ, 401, 584

Ferriere, K. 1998a, ApJ, 497, 759

Ferriere, K. 1998b, A\&A, 335, 488

Ferriere, K. M. 2001, Rev. Mod. Phys, 73, 1031

Ferriere, K. M., \& Schmitt. D. 2000, A\&A, 358, 125

Fields, B. D., Olive, K. A., Casse, M., \& Vangioni-Flam, E. 2001, A\&A, 370, 623

Giacalone, J., \& Jokipii, R. J. 1999, ApJ, 520, 204

Gilmore, G., Gustaffson, B., Edvardson, B., \& Nissen, P. 1992, Nature, 357,379

Grebel, K. 1997, Rev. Mod. Astron., 10, 29

Hanasz, M., \& Lesch, H. 1998, A\&A, 332, 77

Hanasz, M., \& Lesch, H. 2000, ApJ, 543, 235

Hanasz, M., \& Lesch, H. 2001, Space Sci. Rev., 99, 231

Hanasz, M., Otmianowska-Mazur, K., \& Lesch, H. 2002, A\&A, 386, 347

Hanasz, M., \& Lesch, H. 2002, Astrophys. Space Sci., 281, 289

Helou, G., Soifer, B. T., \& Rowan-Robinson, M. 1985, A\&A, 298, L7

Hughes, D. H., Serjeant, S., Dunlop, J., et al. 1998, Nature, 394, 241
Kahn, F. D., \& Brett, L. 1993, MNRAS, 263, 37

Klein, U., Weiland, H., \& Brinks, E. 1991, A\&A, 246, 323

Korpi, M. J., Brandenburg, A., Shukurov, A., \& Tuominen, I. 1999, A\&A, 350, 230

Koyama, K. P., Petre, P., Gotthelf, E. V., et al. 1995, Nature, 378, 255

Kronberg, P. P. 1994, Rep. Prog. Phys., 57, 325

Kulsrud, R., \& Pearce, S. 1969, ApJ, 156, 445

Lanzetta, K. M., Yahata, N., Pascarelle, S., et al. 2002, ApJ, 570, 492

Lesch, H., \& Chiba, M. 1995, A\&A, 297, 305

Lisenfeld, U., Völk, H. J., \& Xu, C. 1996, A\&A, 314, 745

Majewski, S. R. 1993, ARA\&A, 31, 575

Moss, D., Shukurov, A., \& Sokoloff, D. D. 1999, A\&A, 343, 120

Nagamine, K., Fukugita, M., Cen, R., \& Ostriker, J. P. 2001, ApJ, 558, 497

Parizot, E., \& Drury, L. 2000, A\&A, 356, L66

Parker, E. N. 1966, ApJ, 145, 811

Parker, E. N. 1967, ApJ, 149, 535

Parker, E. N. 1992, ApJ, 401, 137

Parker, E. N., \& Jokipii, J. R. 2000, ApJ, 536, 331

Prantzos, N., Casse, M., \& Vangioni-Flam, E. 1993, ApJ, 403, 630

Prantzos, N., \& Casse, M. 1995, ApJS, 92, 575

Ptuskin, V. S. 2001, Space Sci. Rev., 99, 281

Reuter, H.-P., Klein, U., Lesch, H., Wielebinski, R., \& Kronberg, P. P. 1992, A\&A, 282, 724

Reynolds, S. P. 1996, ApJ, 459, L13

Rees, M. 1994, in Cosmical Magnetism, ed. D. Lynden-Bell (Dordrecht: Kluwer), NATO ASI Series C, 422, 155

Schaaf, R., Pietsch, W., Biermann, P. L., et al. 1989, ApJ, 336, 722

Steidel, C. C., Adelberger, K. L., Giavialisco, M., Dickinson, M., \& Pettini, M. 1999, ApJ, 519, 1

Strong, A. W., \& Moskalenko, I. V. 1998, ApJ, 509, 212

Tanimori, T., Hayami, Y., Kamei, S., et al. 1998, ApJ, 497, L25

Tanuma, S., Yokoyama, T., Kudoh, T., \& Shibata, K. 2003, ApJ, 582, 215

Völk, H. J. 1989, A\&A, 218, 67

Völk, H. J., Klein, U., \& Wielebinski, R. 1989, A\&A, 213, L12

Wolfe, A. M., Lanzetta, K. M., \& Oren, A. L. 1992, ApJ, 388, 17

Wunderlich, E., Klein, U., \& Wielebinski, R. 1987, A\&AS, 69, 487

Wunderlich, E., \& Klein, U. 1988, A\&A, 206, 47

Zweibel, E. G. 2003, ApJ, in press [astro-ph/0212559] 